\title{
A shieldless method for cryogenic cold-vapor supply usage: Theory and practice
}

\author{
V.F. Getmanets ${ }^{\text {a,* }}$, R.S. Mikhalchenko ${ }^{\text {a }}$, P.N. Yurchenko ${ }^{\text {a }}$, G.G. Zhun ${ }^{\text {a }}$, H. Stears ${ }^{\text {b }}$ \\ ${ }^{a}$ Special Research \& Development Bureau (SRDB) for Cryogenic Technologies of ILTPh\&E of NAS of Ukraine, 47 Lenin Ave., Kharkov 310164 \\ Ukraine \\ b Orbita Ltd, Kensington, MD, USA
}

Received 20 September 1999; accepted 20 September 1999

\begin{abstract}
We have proven by numerical analysis and experiment that with the use of the SRDB developed shieldless method for cryogenic vapor usage maximum vapor-cold usage is achieved. It is shown that evaporation is decreased in cryovessels and cryostats by using this method equal to 45 times for helium, 5 times for hydrogen and 1.7 times for nitrogen. (C) 2000 Elsevier Science Ltd. All rights reserved.
\end{abstract}

Keywords: Space cryogenic (F); Cryostats (F); Structural materials (A); Thermal conductivity (C)

Some years ago we offered [1] and then experimentally proved [2], a shieldless method of a cold-vapor usage of cryovessels with liquid $\mathrm{He}, \mathrm{Ne}, \mathrm{H}_{2}, \mathrm{~N}_{2}$, and Ar. This method uses the premise that longitudinal heat conduction of a superinsulation blanket is 3-4 orders higher than transverse conduction. This creates an opportunity for the removal of the transverse heat input through the superinsulation by organization of the thermal contact of its layers with a cryovessel plug and neck in places with fixed temperatures. As a result the cold-vapor transported through the neck is used not only for cooling the neck and its plug, but also for cooling all superinsulation layers. It also has been shown [1] that in vessels with liquid or solid $\mathrm{N}_{2}, \mathrm{Ar}, \mathrm{Ne}, \mathrm{H}_{2}, \mathrm{He}$ such a method is effective for volumes approximately up to 600 1. In order to increase the efficiency of the cold-vapor usage the equations which take into account the thermal parameters of the plug, neck and superinsulation and methods of their contact are necessary. It is convenient in vessels with narrow necks to use the radial system of coordinates. This simplifies the task to a two-dimensional problem.

The heat exchange in the superinsulation blanket, neck and plug is described by the two-dimensional equations of heat conduction

${ }^{*}$ Corresponding author.

$$
\begin{gathered}
\frac{1}{r} \frac{\partial}{\partial r}\left(r \lambda_{r}\left(T_{\mathrm{s}}, r, z\right) \frac{\partial T_{\mathrm{s}}}{\partial z}\right)+\frac{\partial}{\partial r}\left(\lambda_{z}\left(T_{\mathrm{s}}, r, z\right) \frac{\partial T}{\partial z}\right)=0, \\
\frac{\mathrm{d}}{\mathrm{d} z}\left(\lambda_{\mathrm{n}}\left(T_{\mathrm{n}}\right) F_{\mathrm{n}}+\lambda_{\mathrm{g}} \cdot F_{\mathrm{g}}\right) \frac{\mathrm{d} T_{\mathrm{n}}}{\mathrm{d} z}+c_{\mathrm{p}} \cdot \stackrel{\circ}{m} \frac{\mathrm{d} T_{\mathrm{n}}}{\mathrm{d} z}= \\
\quad\left(\lambda_{r}\left(T_{\mathrm{n}}\right) \frac{\partial T_{\mathrm{p}}}{\partial r}+\left.\lambda_{r}\left(T_{\mathrm{s}}, r, z\right) \frac{\partial T_{\mathrm{s}}}{\partial r}\right|_{r=R_{N}}\right) \cdot \frac{F_{\mathrm{n}}}{l}, \\
\frac{1}{r} \frac{\partial}{\partial r}\left(r \lambda_{r}\left(T_{\mathrm{p}}\right) \frac{\partial T_{\mathrm{p}}}{\partial z}\right)+\frac{\partial}{\partial z}\left(\lambda_{z}\left(T_{\mathrm{p}}\right) \frac{\partial T_{\mathrm{p}}}{\partial z}\right)=0
\end{gathered}
$$

with boundary conditions of equality of temperatures and heat flows on all surfaces of contact or irradiation.

In the above equations the following notations are used.

$\begin{array}{ll}r, z & \text { radial coordinates } \\ T & \text { temperature } \\ \lambda & \text { heat conductivity coefficient } \\ F_{\mathrm{s}} & \text { section area } \\ l & \text { neck length } \\ r, R & \text { current radius vessels and neck } \\ \text { Subscripts } & \\ \mathrm{n} & \text { neck } \\ \mathrm{p} & \text { plug } \\ \mathrm{s} & \text { superinsulation } \\ \mathrm{g} & \text { gas gap between a plug and neck }\end{array}$


We have a system of nonlinear multidimensional equations of heat conductivity. In order to solve the problem by way of special algorithms of a gradual approximation of convergence, we performed the development by way of imposing physical limits while changing the temperature fields.

From our approach it follows that the maximum efficiency $\varepsilon_{m}=\stackrel{\circ}{m}_{m} / \stackrel{\circ}{m}$ of cryovessels with cold-vapor of usage (where $m_{m}$ is the vapor mass flow rate, when its enthalpy is not used) is represented by the ratio

$k_{r}=\frac{\Delta T_{\Sigma} c_{\mathrm{p}}\left(1-\left(\rho_{\mathrm{v}} / \rho_{\mathrm{L}}\right)\right)}{r}$.

Here $k_{r}$ reflects the ratio between vapor enthalpy and latent heat $(r)$ of the cryogen, and $\Delta T_{\Sigma}$ - the difference between room temperature and the cryogen's boiling point. $\rho_{\mathrm{v}}, \rho_{\mathrm{L}}$ are, respectively, the density of the vapor and the liquid.

Values of $k_{r}$ and $\varepsilon_{m}$ for the various liquids are

$k_{r}=1\left(\mathrm{~N}_{2}\right), 7.8\left(\mathrm{H}_{2}\right), 63(\mathrm{He})$;

$\varepsilon_{m}=1.7\left(\mathrm{~N}_{2}\right), 5.0\left(\mathrm{H}_{2}\right), 45(\mathrm{He})$.

For analytical calculation of the maximum cryovessel efficiency, $\varepsilon_{m}$, with maximal use of the cold-vapor it is possible to use the equation for the vapor mass flow rate from cryovessels

$r \cdot \stackrel{\circ}{m}\left(1-\frac{\rho_{\mathrm{v}}}{\rho_{\mathrm{L}}}\right)=\left.\Lambda^{\Sigma}(T) \frac{\mathrm{d} T_{\mathrm{n}}}{\mathrm{d} z}\right|_{z=0}$,

where $z=0$ is the coordinate $z$ on the cold neck end and $\Lambda^{\Sigma}$ is the generalized heat conductivity of a cryovessels heat protection. $\Lambda^{\Sigma}$ is represented by

$\Lambda^{\Sigma}(T)=\frac{\lambda_{\mathrm{n}} F_{\mathrm{n}}}{l}+\frac{\lambda_{\mathrm{p}} F_{\mathrm{p}}}{l}+\frac{\lambda_{\mathrm{s}} F_{\mathrm{s}}}{\delta_{\mathrm{s}}}$.

Here $\delta_{\mathrm{s}}$ is the thickness of a cryovessel superinsulation blanket.

The temperature dependence of generalized heat conductivity $\Lambda^{\Sigma}$ can be approximated by $\Lambda^{\Sigma}=a \cdot T^{n}$, where $a$ and $n$ are constants. Assuming (for $\mathrm{He}$ and $\mathrm{H}_{2}$ ) $k_{r} \gg 1$, we have an equation for cryovessel maximum efficiency

$\varepsilon_{m}=\frac{k_{r} n}{n+1}$.

The temperature dependence of the heat conductivity $\Lambda^{\Sigma}$ is best described by a parameter of degree, $n=2$. Then for helium and hydrogen, respectively, we find $\varepsilon_{m}=45$ and 5 , as previously stated. For nitrogen the ratio $k_{r} \gg 1$ does not apply. The more complex expression for $\varepsilon_{m}$ does apply and results in the derived value $k_{r}=1.7$. Eqs. (4)-(6) apply for a limiting case when the longitudinal temperature difference along the superinsulation is small. For example, it essentially does not exceed its difference on the neck. It can be shown that in this case the heat inputs to all elements (superinsulation, plug, neck) decrease at the expense of a cold-vapor in identical time and the vapor part continues to cool each element. The cooling rate is proportional to the initial heat input (without coldvapor usage) through the elements, i.e., attitudes

$q_{\mathrm{s}}=\frac{\lambda_{\mathrm{s}} \Delta T_{\Sigma}}{\delta_{\mathrm{s}}}, \quad q_{\mathrm{n}}=\frac{\lambda_{\mathrm{n}} \Delta T_{\Sigma}}{I}, \quad q_{\mathrm{p}}=\frac{\lambda_{\mathrm{p}} \Delta T_{\Sigma}}{I}$.

Now we shall write down the equation of heat conductivity for the heat-protection as a whole for a onedimensional case

$\frac{\mathrm{d}}{\mathrm{d} x}\left(\Lambda^{\Sigma}(T)\right) \frac{\mathrm{d} T}{\mathrm{~d} x}=m c_{\mathrm{p}} \frac{\mathrm{d} T}{\mathrm{~d} x}$.

For simplification of the analysis of heat conductivity we set $\Lambda^{\Sigma}$ constant, and coefficient $K=c_{p} \cdot \Delta T^{\Sigma} / \Lambda^{\Sigma}$ large enough (the condition $K \gg 1$ applies only to helium, hydrogen and neon). Then Eq. (9) is such

$Q^{\Sigma}=Q_{0} \exp (-K) ; \quad Q_{0}=\Lambda^{\Sigma} \Delta T_{\Sigma}$.

In particular, we shall consider the presence of two elements with any distribution of a cold-vapor between them: $\beta_{1}$ is the portion of the first element in total heat input and $f_{1}$ is the portion of vapor applied to the cooling of the first element. In the terms $\beta_{1}$ and $f_{1}$, total heat input are

$$
\begin{aligned}
Q^{\Sigma}= & f_{1} Q_{0} \exp \left(-d_{1} \cdot k\right)+\left(1-f_{1}\right) Q_{0} \\
& \times \exp \left(-\frac{1-d_{1}}{1-f_{1}} k\right) .
\end{aligned}
$$

Differentiating this equation on the parameter $\beta_{1}$ and by equating the differential to zero we obtain the following relationship:

$\exp \left(-\frac{d_{1} k}{f_{1}}\right)=\exp \left(-\frac{1-d_{1}}{1-f_{1}} k\right)$.

From Eq. (12) we see that the condition of a minimum heat input to a vessel is carried out with equality $d_{1}=f_{1}$, i.e.

$d_{1}=f_{1}, \quad Q^{\Sigma}=\Lambda^{\Sigma} \Delta T_{\Sigma} \exp (-K)$.

Thus, in the case of an optimum distribution of the cold-vapor between elements of the heat-protection system (adhering to the laws of proportionality to heat input, $Q_{\mathrm{i}}$ ), total ideal heat input is equal to actual heat input in case that there is ideal contact of adjoining elements in the zone of contact. There is a different type of contact between the heat protection of the system (neck, plug and superinsulation) and the cold-vapor used for cooling of the actual elements. There are different heat inputs through these elements and thus different values of total heat input, $Q_{0}$.

In practice both of these conditions will most likely be broken if the design of the heat-protection of cryovessel is not optimal. As is seen in Fig. 1, on a 


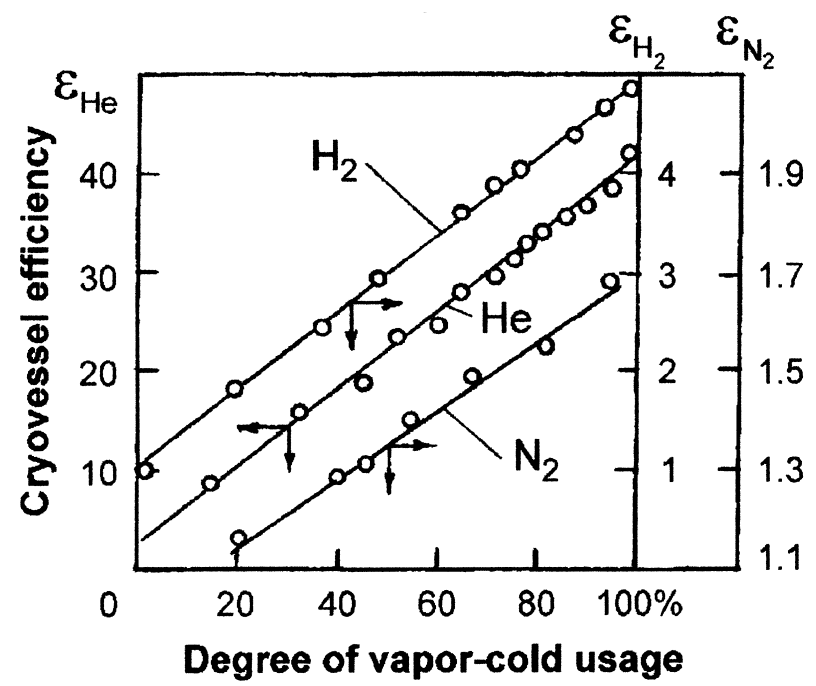

Fig. 1. Influence of vapor-cold usage degree on efficiency using cryobiological vessels (volume 35 1).

typical cryovessel with $\mathrm{N}_{2}, \mathrm{H}_{2}$ and He efficiency of a vessel, $\varepsilon_{m}$, the use of a cold-vapor can be reduced significantly. The experimental data used for Fig. 1 confirms the theoretical data with maximum efficiency for vessels: $\varepsilon_{m}=1,7$ (for nitrogen), $\varepsilon_{m}=5$ (for hydrogen) and $\varepsilon_{m}=45$ (for helium) for the case where we use $100 \%$ cold-vapor.

\section{Conclusion}

1. The numerical and analytical analysis of the maximal increase of cryovessels efficiency, $\varepsilon_{m}$, with $100 \%$ use of the cryogen cold-vapor has been accomplished. These values differ from the value of $k_{r}$ (the relationship of vapor enthalpy to heat of evaporation) which equals 1; 7.8 and 63 accordingly for nitrogen, hydrogen and helium. It has been shown, that the maximum $\varepsilon_{m}$ values are equal to $\varepsilon_{m}=1,7$ (for nitrogen), $\varepsilon_{m}=5$ (for hydrogen) and $\varepsilon_{m}=45$ (for helium).

2. We have validated our technology for blanket design assures the maximal contact of the superinsulation blanket with the neck and high longitudinal heat conductivity of the superinsulation blanket.

3. Experimentally vessels and cryostats with volumes up to 351 realize $100 \%$ use of the cryogenics cold-vapor.

4. It has been shown, that the maximum experimental efficiency of cryovessels with $100 \%$ use of the cold vapor-cold coincides with mathematical analysis and analytical results.

\section{References}

[1] Getmanets VF, Mikhalchenko RS, Yurchenko PN. Soviet Phys J 1982;42:78-86.

[2] Zhun GG, Getmanets VF, Mikhalchenko RS. Soviet Phys J 1988;54:600-7. 